\title{
ONE NON-RELATIVISTIC PARTICLE COUPLED TO A PHOTON FIELD
}

\author{
CHRISTIAN HAINZL ${ }^{1}$
}

\begin{abstract}
We investigate the ground state energy of an electron coupled to a photon field. First, we regard the self-energy of a "free" electron, which we describe by the Pauli-Fierz Hamiltonian. We show that, in the case of small values of the coupling constant $\alpha$, the leading order term is represented by $2 \alpha \pi^{-1}(\Lambda-\ln [1+\Lambda])$.

Next we put the electron in the field of an arbitrary external potential $V$, such that the corresponding Schrödinger operator $p^{2}+V$ has at least one eigenvalue, and show that by coupling to the radiation field the binding energy increases, at least for small enough values of the coupling constant $\alpha$. Moreover, we provide concrete numbers for $\alpha$, the ultraviolet cut-off $\Lambda$, and the radiative correction for which our procedure works.
\end{abstract}

\section{INTRODUCTION AND MAIN RESULTS}

In recent times great effort was made to start a program with the goal to put Quantum Electrodynamics (QED) on a firm mathematical footing. Bach, Fröhlich, and Sigal were the first who proved (in BFS1, BFS2, BFS3]), at least for small values of various parameters, that atoms and molecules have a ground state in the presence of a quantized radiation field. In the one particle case Griesemer, Lieb, and Loss in GLL succeeded in removing the restrictions on the parameters. We refer to GLL for an extensive list of references concerning this subject.

In the present paper we are especially interested in the behavior of one electron coupled to a photon field. We consider the "free" case as well as the presence of an arbitrary external potential $V$ with at least one negative eigenvalue in the electron case. We are interested in the ground state energy of such an electron when the coupling constant $\alpha$ is small and the ultraviolet cutoff parameter $\Lambda$ is fixed.

Without radiation field the self-energy of an electron is simply given by the bottom of the spectrum of $-\Delta$, which is equal to 0 . In the case of coupling to a photon field the situation changes dramatically and the self-energy is a complicated function depending on $\alpha$ and $\Lambda$. The fact that evaluating this quantity is a highly non-trivial problem was pointed out in LL by Lieb and Loss, who, in contrary to us, considered the case of fixed $\alpha$ and large values of the cutoff parameter $\Lambda$.

Date: November 19, 2018.

${ }^{1}$ Marie Curie Fellow 
The paper is organized as follows. In the present section we state our main results on the self-energy and on the binding energy of an electron, which will be proved in Sections 3 and 4. In Section 2 we comment on our notation and in Section 5 we evaluate the radiative correction in the case of a nucleus. We provide concrete numbers for $\alpha$ and $\Lambda$ for which our procedure works.

1.1. Self-energy of an electron. The self-energy of an electron is described by the Pauli-Fierz operator

$$
T=(p+\sqrt{\alpha} A(x))^{2}+\sqrt{\alpha} \sigma B(x)+H_{f} .
$$

We fix units such that $\hbar=c=1$ and the electron mass $m=\frac{1}{2}$. The electron charge is then given by $e=\sqrt{\alpha}$, with $\alpha \approx 1 / 137$ the fine structure constant. In the present paper $\alpha$ plays the role of a small, dimensionless number which measures the coupling to the radiation field. Our results hold for sufficiently small values of $\alpha . \sigma$ is the vector of Pauli matrices $\left(\sigma_{1}, \sigma_{2}, \sigma_{3}\right)$. Recall, the $\sigma_{i}$ are hermitian $2 \times 2$ matrices and fulfill the anticommutation relations $\sigma_{i} \sigma_{j}+\sigma_{j} \sigma_{i}=2 I_{\mathbb{C}^{2}} \delta_{i, j}$. The operator $p=-i \nabla$ is the electron momentum while $A$ is the magnetic vector potential. The magnetic field is $B=\operatorname{curl} A$.

The underlying Hilbert space is

$$
\mathcal{H}=\mathcal{L}^{2}\left(\mathbb{R}^{3} ; \mathbb{C}^{2}\right) \otimes \mathcal{F}_{b}\left(\mathcal{L}^{2}\left(\mathbb{R}^{3} ; \mathbb{C}^{2}\right)\right)
$$

where $\mathcal{F}_{b}$ is the bosonic Fock space for the photon field.

The vector potential is

$$
A(x)=\sum_{\lambda=1,2} \frac{1}{2 \pi} \int_{\mathbb{R}^{3}} \frac{\chi(|k|)}{|k|^{1 / 2}} \varepsilon_{\lambda}(k)\left[a_{\lambda}(k) e^{i k x}+a_{\lambda}^{*}(k) e^{-i k x}\right] d k,
$$

and the corresponding magnetic field reads

$$
B(x)=\sum_{\lambda=1,2} \frac{1}{2 \pi} \int_{\mathbb{R}^{3}} \frac{\chi(|k|)}{|k|^{1 / 2}}\left(k \wedge i \varepsilon_{\lambda}(k)\right)\left[a_{\lambda}(k) e^{i k x}-a_{\lambda}^{*}(k) e^{-i k x}\right] d k,
$$

where the operators $a_{\lambda}$ and $a_{\lambda}^{*}$ satisfy the usual commutation relations

$$
\left[a_{\nu}(k), a_{\lambda}^{*}(q)\right]=\delta(k-q) \delta_{\lambda, \nu}, \quad\left[a_{\lambda}(k), a_{\nu}(q)\right]=0, \quad \text { etc. }
$$

The vectors $\varepsilon_{\lambda}(k) \in \mathbb{R}^{3}$ are any two possible orthonormal polarization vectors perpendicular to $k$ which we assume to be either odd or even, i.e.

$$
\varepsilon_{\lambda}(-k)= \pm \varepsilon_{\lambda}(k) .
$$

The function $\chi(|k|)$ describes the ultraviolet cutoff on the wavenumbers $k$. We choose $\chi$ to be the Heaviside function $\Theta\left(\Lambda-|k| / l_{C}\right)$, where $l_{C}=\hbar /(m c)$ is the Compton wavelength. Therefore $\Lambda=1 / 4$ corresponds to the photon energy $m c^{2}$, which sometimes is considered as a natural upper bound for the cut-off parameter, since it is the maximum value that guarantees that no pair-production takes place. 
The photon field energy $H_{f}$ is given by

$$
H_{f}=\sum_{\lambda=1,2} \int_{\mathbb{R}^{3}}|k| a_{\lambda}^{*}(k) a_{\lambda}(k) d k .
$$

We estimate the leading order in $\alpha$ of the ground state

$$
\Sigma_{\alpha}=\inf \operatorname{spec} T \text {. }
$$

Recall that $\alpha\left\langle 0\left|A^{2}\right| 0\right\rangle=\alpha \pi^{-1} \Lambda^{2}$, where $|0\rangle$ is the vacuum in $\mathcal{F}_{b}$, describes the vacuum fluctuation of the field $A$. Thus, this term is somehow ab initio included in the self-energy. Therefore it may turn out as a slight surprise that $\alpha \pi^{-1} \Lambda^{2}$ is not the leading order term of $\Sigma_{\alpha}$, which relies on the fact that the magnetic field $\sqrt{\alpha} \sigma B$ reproduces the same number but with a negative sign. We show that the leading order term is given by $2 \alpha \pi^{-1}[\Lambda-\ln (1+\Lambda)]$, which is the content of our first theorem.

THEOREM 1. Let $\Lambda$ be fixed but arbitrary. Then

$$
\left|\Sigma_{\alpha}-2 \alpha \pi^{-1}[\Lambda-\ln (1+\Lambda)]\right| \leq C_{1} \alpha^{2}\left(\Lambda^{2}+\Lambda^{3}\right)+C_{2} \alpha^{3}\left(\Lambda^{3}+\Lambda^{4}\right) \ln (1+\Lambda)
$$

for some constants $C_{1,2}>0$ independent of $\alpha$ and $\Lambda$.

It turns out in the proof that one photon is enough to recover the leading order term in $\alpha$.

1.2. Increase of the binding energy. We consider an electron in an arbitrary external potential, described by a real valued function $V$, independent of $\alpha$, which satisfies (i) that the negative part of $V, V_{-}$, is dominated by the kinetic energy $p^{2}=-\Delta$,

$$
V_{-} \leq-\varepsilon \Delta+C_{\varepsilon}
$$

for any $\varepsilon>0$ and for some positive constant $C_{\varepsilon}$, such that the self-adjointness and boundedness from below of $p^{2}+V$ is guaranteed. Additionally, since we want the essential spectrum to start at 0 , we require (ii) that $V_{-}$tends to 0 at infinity. Furthermore, we assume (iii) that the operator $p^{2}+V$ has at least one negative energy bound state.

The corresponding operator with a quantized radiation field reads

$$
\mathbf{H}_{\alpha}=T+V,
$$

for which Hi guarantees self-adjointness. Recently, Griesemer, Lieb, and Loss GLL proved for all values of $\alpha, \Lambda$ that $\mathbf{H}_{\alpha}$ has an eigenstate, as well as that the binding energy $\Sigma_{\alpha}-\mathbf{E}_{\alpha}$, with

$$
\mathbf{E}_{\alpha}=\inf \operatorname{spec} \mathbf{H}_{\alpha},
$$

cannot decrease, i.e.

$$
\mathbf{E}_{\alpha}-\Sigma_{\alpha} \leq-e_{0}
$$

where $-e_{0}=\inf \operatorname{spec}\left[p^{2}+V\right]$. We show that at least for small values of the coupling constant $\alpha$ the binding energy increases, namely (1.11) holds with strict inequality. 
THEOREM 2. Let $V$ satisfy the conditions (i)-(iii), and $\phi$ be the ground state of $p^{2}+V$ with corresponding energy $-e_{0}$. Then

$$
\mathbf{E}_{\alpha}-\Sigma_{\alpha} \leq-e_{0}-\alpha \mathcal{E}(V, \Lambda)+\mathcal{O}\left(\alpha^{2}\right)
$$

for some positive number $\mathcal{E}(V, \Lambda)$ depending on $V$ and the cut-off $\Lambda$. Moreover, there exists a number $\rho>0$, such that for all $\alpha \in(0, \rho]$

$$
\mathbf{E}_{\alpha}-\Sigma_{\alpha}<-e_{0} .
$$

REMARK 1. The equation (1.12) holds for all real $V$, such that $p^{2}+V$ is self-adjoint and has a ground state. But only for $V$ satisfying $(i)-(i i i)$ we know that $H_{\alpha}$ has a ground state.

We actually check that $\mathcal{E}(V, \Lambda)$ is bounded from above by

$$
\mathcal{E}(V, \Lambda) \leq\|p \phi\|^{2} \frac{32 \pi}{3} \ln [1+\Lambda]
$$

In the spinless case $(B=0)$ it was previously proven ( $[\mathrm{HVV}]$ ) that coupling to a photon field can produce a ground state even if the corresponding Schrödinger operator has only continuous spectrum. This phenomenon of enhanced binding was earlier shown in [HS] in the case of dipole approximation $(A=A(0))$ in the limit of large values of the coupling constant $\alpha$.

\section{NotATiOn}

Throughout the paper we use the notation

$$
A(x)=D(x)+D^{*}(x), B(x)=E(x)+E^{*}(x)
$$

for the vector potential, respectively the magnetic field.

The operators $D^{*}$ and $E^{*}$ create a photon with wave functions $G(k) e^{-i k \cdot x}$ and $H(k) e^{-i k \cdot x}$, respectively, where $G(k)=\left(G^{1}(k), G^{2}(k)\right)$ and $H(k)=$ $\left(H^{1}(k), H^{2}(k)\right)$ are vectors of one-photon states, given by

$$
G^{\lambda}(k)=\frac{\chi(|k|)}{2 \pi|k|^{1 / 2}} \varepsilon_{\lambda}(k) .
$$

and

$$
H^{\lambda}(k)=\frac{-i \chi(|k|)}{2 \pi|k|^{1 / 2}} k \wedge \varepsilon_{\lambda}(k)
$$

Applying Fourier transform, with respect to $k$, on $G(k) e^{-i k \cdot x}$, i.e.

$$
\mathcal{F}^{-1}\left[G(k) e^{-i k \cdot x}\right](\xi)=\int_{\mathbb{R}^{3}} e^{i k \cdot(\xi-x)} G(k) d k \equiv D^{*}|0\rangle(\xi-x),
$$

we see that $D^{*}$ creates a photon depending on $\xi-x$, where $\xi$ denotes the photon variable in configuration space.

If the reader is not used in thinking of a "position" of a photon, he may consider it as a simple unitary transform. Because, now we introduce the 
variable $\eta=\xi-x$ and the object we are going to work with is the Fourier transform with respect to $\eta$, namely

$$
\mathcal{F}\left[D^{*}|0\rangle(\eta)\right](k)=G(k) .
$$

The same result holds for $E^{*}$ if $G(k)$ is replaced by $H(k)$.

Notice that in the so chosen notation the electron momentum $p=-i \nabla_{x}$ of a one photon state $D^{*} f(x, \eta)=f(x) \otimes D^{*}|0\rangle(\eta)$, with $\eta=\xi-x$, can be written as

$$
\begin{aligned}
p D^{*} f(x, \eta)=\sum_{i=1}^{3}\left(p_{x}^{i} f(x)\right) \otimes D_{i}^{*}|0\rangle(\eta)-f(x) & \otimes\left(p_{\eta} D^{*}|0\rangle(\eta)\right) \\
& =\left[p_{x}-p_{\eta}\right] D^{*} f(x, \eta) .
\end{aligned}
$$

It turns out to be convenient to denote a general vector $\Psi \in \mathcal{H}$ as a sequence

$$
\Psi=\left\{\psi_{0}, \psi_{1}, \psi_{2}, \ldots\right\}
$$

where

$$
\psi_{n}=\psi_{n}\left(x, \xi_{1}-x, \ldots, \xi_{n}-x\right),
$$

with relative variables $\xi_{i}-x=\eta_{i}$. For simplicity, we suppress the polarization of the photons and the spin of the electron.

\section{Proof of Theorem 1}

3.1. Upper bound. We choose the sequence of trial wave functions

$$
\Psi_{n}=\left\{f_{n}(x) \uparrow,-\sqrt{\alpha}\left[p_{\eta}^{2}+H_{f}\right]^{-1}(\sigma \uparrow) E^{*} f_{n}(x, \eta), 0,0, \ldots\right\},
$$

with $\eta=\xi-x, f_{n} \in \mathcal{L}^{2}\left(\mathbb{R}^{3}\right),\left\|f_{n}\right\|=1$ and $\uparrow$ denoting the spin-up vector in $\mathbb{C}^{2}$. We assume for the electron part $f_{n}(x)$ that $\left\|p f_{n}\right\| \rightarrow 0$ as $n \rightarrow \infty$, which means that the electron spreads out to infinity. This is reasonable, for in the case without coupling this sequence recovers the bottom of the spectrum of $-\Delta$. Recall,

$$
E^{*} f_{n}(x, \eta) \equiv f_{n}(x) \otimes\left[E^{*}|0\rangle\right](\eta)
$$

and, due to $\eta=\xi-x$,

$$
\left(\left[p^{2}+H_{f}\right] E^{*} f_{n}\right)(x, \eta)=\left(\left[p_{x}-p_{\eta}\right]^{2}+\left|p_{\eta}\right|\right) E^{*} f_{n}(x, \eta) .
$$

Notice, in the one photon case $H_{f}=\left|p_{\eta}\right|$. Observe,

$$
\begin{aligned}
{[p, D]=\sum_{\lambda=1,2} \int_{\mathbb{R}^{3}} G^{\lambda}(k) \cdot\left[p, e^{i k \cdot x}\right] a_{\lambda}(k) d k=} \\
=\sum_{\lambda=1,2} \int_{\mathbb{R}^{3}}\left(G^{\lambda}(k) \cdot k\right) e^{i k \cdot x} a_{\lambda}(k) d k=0
\end{aligned}
$$

by our choice of $\varepsilon_{\lambda}(k)$. Analogously,

$$
\left[p, D^{*}\right]=[p, E]=\left[p, E^{*}\right]=0 .
$$


By means of Schwarz' inequality it is easy to see that by our assumption $\left\|p f_{n}\right\| \rightarrow 0$

$$
\lim _{n \rightarrow \infty}\left(\Psi_{n}, p A \Psi_{n}\right)=2 \lim _{n \rightarrow \infty} \Re\left(\Psi_{n}, p D \Psi_{n}\right)=0 .
$$

Similarly, if we denote the 1-photon part of $\Psi$ as

$$
\psi_{1}^{n}=-\sqrt{\alpha}\left[p_{\eta}^{2}+H_{f}\right]^{-1}(\sigma \uparrow) E^{*} f_{n},
$$

then

$$
\left(\psi_{1}^{n}, p^{2} \psi_{1}^{n}\right)=\left(\psi_{1}^{n},\left[p_{x}+p_{\eta}\right]^{2} \psi_{1}^{n}\right)=\left(\psi_{1}^{n}, p_{\eta}^{2} \psi_{1}^{n}\right)+\operatorname{Error}\left(p_{x} f_{n}\right),
$$

with $\lim _{n \rightarrow \infty} \operatorname{Error}\left(p_{x} f_{n}\right)=0$. Furthermore, we compute

$$
\begin{aligned}
\left(\psi_{1}^{n},\left[p_{\eta}^{2}+H_{f}\right] \psi_{1}^{n}\right)+2 \sqrt{\alpha} \Re\left((\sigma \uparrow) E^{*} f_{n}, \psi_{1}^{n}\right) & \\
=-\alpha\left((\sigma \uparrow) E^{*}\right. & \left.f_{n},\left[p_{\eta}^{2}+H_{f}\right]^{-1}(\sigma \uparrow) E^{*} f_{n}\right) \\
& =-\alpha\left\langle 0\left|(\sigma \uparrow) E\left[p^{2}+H_{f}\right]^{-1}(\sigma \uparrow) E^{*}\right| 0\right\rangle
\end{aligned}
$$

In momentum representation, recall $\mathcal{F}\left[E^{*}|0\rangle\right](k)=H(k)$, the last term in (3.9) equals

$$
\begin{aligned}
\left\langle 0\left|(\sigma \uparrow) E\left[p^{2}+H_{f}\right]^{-1}(\sigma \uparrow) E^{*}\right| 0\right\rangle=\int \frac{|H(k) \cdot(\sigma \uparrow)|_{\mathbb{C}^{2}}^{2}}{|k|^{2}+|k|} d k \\
=\int \frac{|H(k)|^{2}}{|k|^{2}+|k|} d k=\left\langle 0\left|E\left[p^{2}+H_{f}\right]^{-1} E^{*}\right| 0\right\rangle .
\end{aligned}
$$

The equality from first to second line holds, because of the anti-commutation relations of $\sigma$ and the fact that $H$ is purely imaginary.

For simplicity, we will use throughout the paper

$$
|H(k)|^{2}=\sum_{\lambda=1,2}\left|H^{\lambda}(k)\right|^{2}, \quad|G(k)|^{2}=\sum_{\lambda=1,2}\left|G^{\lambda}(k)\right|^{2} .
$$

We evaluate

$$
\begin{aligned}
\left\langle 0\left|E\left[p^{2}+H_{f}\right]^{-1} E^{*}\right| 0\right\rangle & =\sum_{\lambda=1,2} \int \frac{\left|H^{\lambda}(k)\right|^{2}}{|k|^{2}+|k|} d k \\
& =\frac{1}{2 \pi^{2}} \int \frac{|k| \chi(|k|)}{|k|^{2}+|k|} d k=\pi^{-1}\left[\Lambda^{2}-2[\Lambda-\ln (1+\Lambda)]\right] .
\end{aligned}
$$

Since

$$
\left[D, D^{*}\right] \equiv D D^{*}-D^{*} D=\pi^{-1} \Lambda^{2}
$$

we know

$$
A^{2}=\left(D+D^{*}\right)^{2}=\pi^{-1} \Lambda^{2}+2 D^{*} D+D^{*} D^{*}+D D .
$$

With $\left(\Psi_{n}, D D \Psi_{n}\right)=0$ and $\lim _{n \rightarrow \infty}\left(\psi_{1}^{n}, \psi_{1}^{n}\right) \leq 2 \alpha \pi^{-1} \ln (1+\Lambda)$ we arrive at

$$
\begin{array}{r}
\lim _{n \rightarrow \infty}\left(\Psi_{n}, T \Psi_{n}\right) \leq \alpha \pi^{-1} \Lambda^{2}-\alpha\left\langle 0\left|E\left[p^{2}+H_{f}\right]^{-1} E^{*}\right| 0\right\rangle+2 \pi^{-2} \alpha^{2} \Lambda^{2} \ln (1+\Lambda) \\
+2 \alpha^{2} \| D\left[p^{2}+H_{f}\right]^{-1}(\sigma \uparrow) E^{*}|0\rangle \|^{2} .
\end{array}
$$


The last term in the r.h.s. vanishes which can be seen by explicit calculations using the relation

$$
\sum_{\lambda=1,2} \varepsilon_{\lambda}^{i} \varepsilon_{\lambda}^{j}=\delta_{i, j}-\frac{k_{i} k_{j}}{|k|^{2}} .
$$

Namely, if $\epsilon^{j l n}$ denotes the totally antisymmetric epsilon-tensor, we obtain

$$
\begin{aligned}
\sum_{\lambda=1,2} \int \frac{G_{i}^{\lambda}(k) H_{j}^{\lambda}(k)}{|k|^{2}+|k|} d k & =\sum_{\lambda=1,2} \sum_{l, n=1}^{3} i \int \frac{\chi(|k|) \varepsilon_{\lambda}^{i}(k)\left[\epsilon^{j l n} \varepsilon_{\lambda}^{l}(k) k_{n}\right]}{|k|^{3}+|k|^{2}} d k \\
& =\sum_{l, n=1}^{3} i \int \frac{\chi(|k|)\left[\delta_{i, l}-\frac{k_{i} k_{l}}{|k|^{2}}\right] \epsilon^{j l n} k_{n}}{|k|^{3}+|k|^{2}} d k=0 .
\end{aligned}
$$

Therefore, since $\left(\Psi_{n}, \Psi_{n}\right)=1+$ const. $\alpha$, we obtain the upper bound

$$
\begin{aligned}
& \Sigma_{\alpha} \leq \lim _{n \rightarrow \infty}\left(\Psi_{n}, T \Psi_{n}\right) /\left(\Psi_{n}, \Psi_{n}\right) \leq \lim _{n \rightarrow \infty}\left(\Psi_{n}, T \Psi_{n}\right) \leq \\
& \leq 2 \alpha \pi^{-1}[\Lambda-\ln (1+\Lambda)]+2 \pi^{-2} \alpha^{2} \Lambda^{2} \ln (1+\Lambda) .
\end{aligned}
$$

3.2. Lower bound. We have learned in the previous section that an approximate ground state $\Psi,\|\Psi\|=1$, satisfies

$$
(\Psi, T \Psi) \leq 2 \alpha \pi^{-1} \Lambda+2 \pi^{-2} \alpha^{2} \Lambda^{2} \ln (1+\Lambda) .
$$

Since

$$
T=[\sigma \cdot(p+\sqrt{\alpha} A(x))]^{2}+H_{f} \geq H_{f}
$$

we immediately get

$$
\left(\Psi, H_{f} \Psi\right) \leq 2 \alpha \pi^{-1} \Lambda+2 \pi^{-2} \alpha^{2} \Lambda^{2} \ln (1+\Lambda) .
$$

Therefore, by means of Schwarz' inequality we obtain

$$
\begin{aligned}
2 \sqrt{\alpha}(\Psi, p A \Psi) & =4 \sqrt{\alpha} \Re(\Psi, p D \Psi) \leq a\|p \Psi\|^{2}+4 a^{-1} \alpha\|D \Psi\|^{2} \\
\sqrt{\alpha}(\Psi, \sigma B \Psi) & =2 \sqrt{\alpha}(\Psi, \sigma E \Psi) \leq c \Lambda^{2} \alpha\|\Psi\|^{2}+c^{-1}\left(1 / \Lambda^{2}\right)\|E \Psi\|^{2},
\end{aligned}
$$

for any $a, c>0$. Since, by $A^{2} \geq 0$,

$$
\|p \Psi\|^{2} \leq(\Psi, T \Psi)-\left(\Psi, H_{f} \Psi\right)-4 \sqrt{\alpha} \Re(\Psi, p D \Psi)-2 \sqrt{\alpha}(\Psi, \sigma E \Psi),
$$

we obtain by (3.22), (3.23), the operator inequalities (e.g. GLL Lemma A. 4])

and (3.21)

$$
D^{*} D \leq \frac{2}{\pi} \Lambda H_{f}, \quad E^{*} E \leq \frac{2}{3 \pi} \Lambda^{3} H_{f},
$$

$$
\|p \Psi\|^{2} \leq C_{1} \alpha\left(\Lambda+\Lambda^{2}\right)+C_{2} \alpha^{2}\left(\Lambda^{2}+\Lambda^{3}\right) \ln (1+\Lambda)
$$

for some suitable constants $C_{1,2}>0$. Equations (3.21) and (3.26) will be decisive to control our error estimates. Recall,

$$
A^{2}=\pi^{-1} \Lambda^{2}+2 D^{*} D+2 \Re D D .
$$


Thus, since $D^{*} D \geq 0$,

$$
(\Psi, T \Psi) \geq \alpha \pi^{-1} \Lambda^{2}\|\Psi\|^{2}+\mathcal{E}_{0}\left[\psi_{0}, \psi_{1}\right]+\sum_{n=0}^{\infty} \mathcal{E}\left[\psi_{n}, \psi_{n+1}, \psi_{n+2}\right],
$$

where

$$
\mathcal{E}_{0}\left[\psi_{0}, \psi_{1}\right]=\left(\psi_{1}, \mathcal{A} \psi_{1}\right)+2 \sqrt{\alpha} \Re\left(\left[\sigma E^{*}+2 p D^{*}\right] \psi_{0}, \psi_{1}\right)
$$

and

$$
\begin{aligned}
& \mathcal{E}\left[\psi_{n}, \psi_{n+1}, \psi_{n+2}\right]=\left(\psi_{n+2}, \mathcal{A} \psi_{n+2}\right) \\
& +2 \Re\left(\left(\left[\sqrt{\alpha} \sigma E^{*}+2 \sqrt{\alpha} p D^{*}\right] \psi_{n+1}+\alpha D^{*} D^{*} \psi_{n}\right), \psi_{n+2}\right),
\end{aligned}
$$

with

$$
\mathcal{A}=p^{2}+H_{f}
$$

Recall, we can write our approximate ground state $\Psi$ as

$$
\Psi=\left\{\psi_{0}(x), \psi_{1}\left(x, \eta_{1}\right), \ldots, \psi_{n}\left(x, \eta_{1}, \ldots, \eta_{n}\right), \ldots\right\},
$$

with $\eta_{i}=\xi_{i}-x$. For convenience we will work in momentum representation

$$
\mathcal{F}\left[\psi_{n}\left(x, \eta_{1}, \ldots, \eta_{n}\right)\right]\left(l, k_{1}, \ldots, k_{n}\right)=\psi_{n}\left(l, k_{1}, \ldots, k_{n}\right),
$$

with $k=\left(k_{1}, \ldots, k_{n}\right)$ and $l$ denotes the momentum representation of the electron variable.

We consider the term $\mathcal{E}\left[\psi_{n}, \psi_{n+1}, \psi_{n+2}\right]$. As a straightforward consequence of Schwarz' inequality we derive

$$
\begin{aligned}
& \mathcal{E}\left[\psi_{n}, \psi_{n+1}, \psi_{n+2}\right] \geq \\
& \quad \geq-\left\|\sqrt{\alpha}\left[\mathcal{A}^{-1 / 2} \sigma E^{*}+\mathcal{A}^{-1 / 2} p D^{*}\right] \psi_{n+1}+\alpha \mathcal{A}^{-1 / 2} D^{*} D^{*} \psi_{n}\right\|^{2}
\end{aligned}
$$

Similarly,

$$
\mathcal{E}_{0}\left[\psi_{0}, \psi_{1}\right] \geq-\alpha\left\|\left(\mathcal{A}^{-1 / 2} \sigma E^{*}+2 \mathcal{A}^{-1 / 2} p D^{*}\right) \psi_{0}\right\|^{2}
$$

The fact that $H(k)$ is purely imaginary and $\mathcal{A}$ commutes with the reflection $l \rightarrow-l$ imply

$$
\Im\left(E_{i}^{*} \psi_{0}, \mathcal{A}^{-1} E_{j}^{*} \psi_{0}\right)=0 .
$$

Together with the anti-commutation relations for $\sigma$ we infer

$$
\left(\sigma E^{*} \psi_{0}, \mathcal{A}^{-1} \sigma E^{*} \psi_{0}\right)=\left(\psi_{0}, E \mathcal{A}^{-1} E^{*} \psi_{0}\right)
$$

Now we are going to evaluate the r.h.s. of (3.34). These evaluations then are also applied to (3.35). First, we consider the diagonal terms. The most important one, that reproduces the leading order in $\alpha$, is

$$
-\alpha\left(\sigma E^{*} \psi_{n+1}, \mathcal{A}^{-1} \sigma E^{*} \psi_{n+1}\right) .
$$


Explicitly, in Fourier representation,

$$
\mathcal{F}\left[E^{*} \psi_{n+1}\right]\left(l, k_{1}, \ldots, k_{n+2}\right)=\frac{1}{\sqrt{n+2}} \sum_{i=1}^{n+2} H\left(k_{i}\right) \psi_{n+1}\left(l, k_{1}, \ldots, k_{i}, \ldots, k_{n+2}\right),
$$

where $\not k_{i}$ indicates that the $i$-th variable is omitted in $\psi_{n+1}$. Due to permutational symmetry the expression (3.38) consists of two different terms

$$
-\alpha\left(\sigma E^{*} \psi_{n+1}, \mathcal{A}^{-1} \sigma E^{*} \psi_{n+1}\right)=-\alpha\left(I_{n+1}+I I_{n+1}\right) .
$$

The diagonal term $I_{n+1}$ where in the right as well as left hand side of (3.38) a photon is created with index $i$ and the mixed term $I I_{n+1}$. By similar arguments as for (3.35), namely if we take advantage of the fact that $H$ is purely imaginary and use the anti-commutation relations of $\sigma$ we obtain

$$
I_{n+1} \leq \int \frac{\left|H\left(k_{n+2}\right)\right|^{2}\left|\psi_{n+1}\left(l, k_{1}, \ldots, k_{n+1}\right)\right|^{2}}{\left[l-\sum_{i=1}^{n+2} k_{i}\right]^{2}+\sum_{i=1}^{n+2}\left|k_{i}\right|} d l d k_{1} \ldots d k_{n+2} .
$$

We set $\mathcal{Q}=\left[l-\sum_{i=1}^{n+1} k_{i}\right]^{2}+\left|k_{n+2}\right|^{2}+\sum_{i=1}^{n+2}\left|k_{i}\right|$ and $b=2\left[l-\sum_{i=1}^{n+1} k_{i}\right] \cdot k_{n+2}$ and expand

$$
\frac{1}{\mathcal{Q}-b}=\frac{1}{\mathcal{Q}}+\frac{1}{\mathcal{Q}} b \frac{1}{\mathcal{Q}}+\frac{1}{\mathcal{Q}} b \frac{1}{\mathcal{Q}-b} b \frac{1}{\mathcal{Q}} .
$$

Plugging this expansion into (3.41), the second term, when integrating over $k_{n+2}$, obviously vanishes. Since $\mathcal{Q} \geq\left|k_{n+2}\right|^{2}+\left|k_{n+2}\right|$ and $\mathcal{Q}-b \geq\left|k_{n+2}\right|$ we estimate

$$
\begin{aligned}
I_{n+1} & \leq\left\|\psi_{n+1}\right\|^{2} \int_{\mathbb{R}^{3}} \frac{|H(k)|^{2}}{|k|^{2}+|k|} d k \\
& +4 \int \frac{\left|H\left(k_{n+2}\right)\right|^{2}\left|k_{n+2}\right|^{2}\left|l-\sum_{i=1}^{n+1} k_{i}\right|^{2}\left|\psi_{n+1}\left(l, k_{1}, \ldots, k_{n+1}\right)\right|^{2}}{\left[\left|k_{n+2}\right|^{2}+\left|k_{n+2}\right|\right]^{2}\left|k_{n+2}\right|} \\
& \leq\left\|\psi_{n+1}\right\|^{2}\left\langle 0\left|E \mathcal{A}^{-1} E^{*}\right| 0\right\rangle+\text { const. } \Lambda\left\|p \psi_{n+1}\right\|^{2} .
\end{aligned}
$$

For the mixed term we have

$$
\begin{aligned}
& I I_{n+1} \leq 3(n+1) \sum_{\lambda=1,2} \int \frac{\left|H^{\lambda}\left(k_{1}\right)\right|\left|H^{\lambda}\left(k_{n+2}\right)\right|}{\left[l-\sum_{i=1}^{n+2} k_{i}\right]^{2}+\sum_{i=1}^{n+2}\left|k_{i}\right|} \times \\
& \quad \times\left|\psi_{n+1}\left(l, k_{2}, \ldots, k_{n+2}\right)\right|\left|\psi_{n+1}\left(l, k_{1}, \ldots, k_{n+1}\right)\right| d l d k_{1} \ldots d k_{n+2} .
\end{aligned}
$$

By means of the one-photon density

$$
\rho_{\psi_{n+1}}(k)=(n+1) \int\left|\psi_{n+1}\left(l, k, k_{2}, \ldots, k_{n+1}\right)\right|^{2} d l d k_{2} \ldots d k_{n+1}
$$


we infer after applying Schwarz' inequality to (3.44)

$$
\begin{aligned}
& I I_{n+1} \leq 3(n+1) \sum_{\lambda=1,2} \int \frac{\left|H^{\lambda}\left(k_{1}\right)\right|\left|H^{\lambda}\left(k_{n+2}\right)\right|}{\left|k_{1}\right|+\left|k_{n+2}\right|} \times \\
& \quad \times \sqrt{\rho_{\psi_{n+1}}\left(k_{1}\right)} \sqrt{\rho_{\psi_{n+1}}\left(k_{n+2}\right)} d k_{1} d k_{n+2} \\
& \leq \frac{6(n+1)}{(2 \pi)^{2}} \int \frac{\chi\left(\left|k_{1}\right|\right) \chi\left(\left|k_{n+2}\right|\right)}{\left|k_{1}\right|+\left|k_{n+2}\right|} \sqrt{\left|k_{1}\right| \rho_{\psi_{n+1}}\left(k_{1}\right)} \times \\
& \times \sqrt{\left|k_{n+2}\right| \rho_{\psi_{n+1}}\left(k_{n+2}\right)} d k_{1} d k_{n+2} \\
& \leq \frac{3}{2 \pi^{2}} \int|k| \rho_{\psi_{n+1}}(k) d k\left[\int \frac{\chi\left(\left|k_{1}\right|\right) \chi\left(\left|k_{n+2}\right|\right)}{\left(\left|k_{1}\right|+\left|k_{n+2}\right|\right)^{2}} d k_{1} d k_{n+2}\right]^{1 / 2} \\
& \leq \text { const. } \Lambda^{2}\left(\psi_{n+1}, H_{f} \psi_{n+1}\right) .
\end{aligned}
$$

Therefore, we summarize

$$
\begin{aligned}
-\alpha\left(\sigma E^{*} \psi_{n+1}, \mathcal{A}^{-1} \sigma E^{*} \psi_{n+1}\right) & \geq-\alpha\left\langle 0\left|E \mathcal{A}^{-1} E^{*}\right| 0\right\rangle\left\|\psi_{n+1}\right\|^{2} \\
& - \text { const. } \alpha \Lambda\left(\left\|p \psi_{n+1}\right\|+\Lambda\left(\psi_{n+1}, H_{f} \psi_{n+1}\right)\right) .
\end{aligned}
$$

All other terms in (3.34) are of order $\mathcal{O}\left(\alpha^{2}\right)$ or even of higher order.

For the second diagonal term we obtain

$$
-\alpha^{2}\left(\psi_{n}, D D \mathcal{A}^{-1} D^{*} D^{*} \psi_{n}\right) \geq- \text { const. } \alpha^{2} \Lambda\left(\Lambda\left\|\psi_{n}\right\|^{2}+\left\|p \psi_{n}\right\|^{2}+\left(\psi_{n}, H_{f} \psi_{n}\right)\right) .
$$

The proof of (3.48), whose strategy is similar to the one for (3.47), will be postponed to Lemma 1 in the appendix.

The third diagonal term of (3.34) reads

$$
\begin{aligned}
& -\alpha\left(p D^{*} \psi_{n+1}, \mathcal{A}^{-1} p D^{*} \psi_{n+1}\right)=\sum_{\lambda=1,2}-\alpha \times \\
& \times\left[\int \frac{\left[G^{\lambda}\left(k_{n+2}\right) \cdot\left(l-\sum_{i=1}^{n+1} k_{i}\right)\right]^{2}\left|\psi_{n+1}\left(l, k_{1}, \ldots, k_{n+1}\right)\right|^{2}}{\left|l-\sum_{i=1}^{n+2} k_{i}\right|^{2}+\sum_{i=1}^{n+2}\left|k_{i}\right|} d l d k_{1} \ldots d k_{n+2}\right. \\
& +(n+1) \int \frac{\left[G^{\lambda}\left(k_{1}\right) \cdot\left(l-\sum_{i=1}^{n+2} k_{i}\right)\right]\left[G^{\lambda}\left(k_{n+2}\right) \cdot\left(l-\sum_{i=1}^{n+2} k_{i}\right)\right]}{\left|l-\sum_{i=1}^{n+2} k_{i}\right|^{2}+\sum_{i=1}^{n+2}\left|k_{i}\right|} \times \\
& \left.\times \frac{\psi_{n+1}\left(l, k_{1}, \ldots, k_{n+1}\right)}{\mid} \psi_{n+1}\left(l, k_{2}, \ldots, k_{n+2}\right) d l d k_{1} \ldots d k_{n+2}\right] \\
& \geq- \text { const. } \alpha \Lambda\left(\left\|p \psi_{n+1}\right\|^{2}+\left(\psi_{n+1}, H_{f} \psi_{n+1}\right)\right) .
\end{aligned}
$$


For the second term in the r.h.s. we used first

$$
\frac{\left|l-\sum_{i=1}^{n+2} k_{i}\right|^{2}}{\left|l-\sum_{i=1}^{n+2} k_{i}\right|^{2}+\sum_{i=1}^{n+2}\left|k_{i}\right|} \leq 1,
$$

and afterwards applied Schwarz' inequality to bound it from below by

$$
\begin{gathered}
\geq-(n+1) \sum_{\lambda=1,2} \int\left|G^{\lambda}\left(k_{1}\right)\right|\left|G^{\lambda}\left(k_{n+2}\right)\right| \sqrt{\rho_{\psi_{n+1}}\left(k_{1}\right) \rho_{\psi_{n+1}}\left(k_{n+2}\right)} d k_{1} d k_{n+2} \\
\geq \text { - const. } \int|k| \rho_{\psi_{n+1}}(k) d k\left[\int \frac{\chi\left(\left|k_{1}\right|\right) \chi\left(\left|k_{n+2}\right|\right)}{\left|k_{1}\right|^{2}\left|k_{n+2}\right|^{2}} d k_{1} d k_{n+2}\right]^{1 / 2}, \quad \text { (3.51) }
\end{gathered}
$$

which yields the last term in the last line of (3.49).

Next we consider the off-diagonal terms. Notice,

$$
\begin{aligned}
& \alpha \Re\left(p D^{*} \psi_{n+1}, \mathcal{A}^{-1} \sigma E^{*} \psi_{n+1}\right)= \\
& =\sum_{\lambda=1,2} \alpha\left[\Re \int \frac{\left[G^{\lambda}\left(k_{n+2}\right) \cdot\left(l-\sum_{i=1}^{n+1} k_{i}\right)\right] H^{\lambda}\left(k_{n+2}\right)}{\left|l-\sum_{i=1}^{n+2} k_{i}\right|^{2}+\sum_{i=1}^{n+2}\left|k_{i}\right|} \times\right. \\
& \quad \times \cdot\left\langle\psi_{n+1}, \sigma \psi_{n+1}\right\rangle_{\mathbb{C}^{2}}\left(l, k_{1}, \ldots, k_{n+1}\right) d l d k_{1} \ldots d k_{n+2} \\
& +(n+1) \Re \int \frac{\left[G^{\lambda}\left(k_{1}\right) \cdot\left(l-\sum_{i=2}^{n+2} k_{i}\right)\right] H^{\lambda}\left(k_{n+2}\right)}{\left|l-\sum_{i=1}^{n+2} k_{i}\right|^{2}+\sum_{i=1}^{n+2}\left|k_{i}\right|} \times \\
& \left.\times \cdot\left\langle\psi_{n+1}\left(l, k_{2}, \ldots, k_{n+2}\right), \sigma \psi_{n+1}\left(l, k_{1}, \ldots, k_{n+1}\right)\right\rangle_{\mathbb{C}^{2}} d l d k_{1} \ldots d k_{n+2}\right] .
\end{aligned}
$$

The first term in the r.h.s. vanishes, because the integral is purely imaginary. For the second term we use $\frac{|a|}{a^{2}+b} \leq \frac{1}{2} b^{-1 / 2}$ and Schwarz' inequality to bound it from above by

$$
\begin{array}{r}
\text { (3.52) } \leq \alpha \sum_{\lambda=1,2} \int \frac{\left|G^{\lambda}\left(k_{1}\right)\right|\left|H^{\lambda}\left(k_{n+2}\right)\right|}{\left(\left|k_{1}\right|+\left|k_{n+2}\right|\right)^{1 / 2}\left|k_{1}\right|^{1 / 2}\left|k_{n+2}\right|^{1 / 2}} \times \\
\quad \times \sqrt{\rho_{\psi_{n+1}}\left(k_{1}\right)\left|k_{1}\right|} \sqrt{\rho_{\psi_{n+1}}\left(k_{n+2}\right)\left|k_{n+2}\right|} d k_{1} d k_{n+2} \\
\leq \text { const. } \alpha \Lambda^{3 / 2}\left(\psi_{n+1}, H_{f} \psi_{n+1}\right) .
\end{array}
$$

The second off-diagonal term, $2 \alpha^{3 / 2} \Re\left(p D^{*} \psi_{n+1}, \mathcal{A}^{-1} D^{*} D^{*} \psi_{n}\right)$, can simply be bounded from above by

$$
\alpha\left(\psi_{n+1}, p D \mathcal{A}^{-1} p D^{*} \psi_{n+1}\right)+\alpha^{2}\left(\psi_{n}, D D \mathcal{A}^{-1} D^{*} D^{*} \psi_{n}\right),
$$

on which we now apply (3.48) and (3.49). 
Finally, by means of Lemma 2 in the appendix we estimate the last offdiagonal term by

$$
\alpha^{3 / 2} \Re\left(\sigma E^{*} \psi_{n+1}, \mathcal{A}^{-1} D^{*} D^{*} \psi_{n}\right) \leq \text { const. } \alpha \Lambda\left(\alpha\left\|\psi_{n+1}\right\|^{2}+\left(\psi_{n}, H_{f} \psi_{n}\right)\right) .
$$

Collecting above estimates and summing over all $n$ we arrive at

$$
\begin{array}{r}
(\Psi, T \Psi) \geq 2 \alpha \pi^{-1}(1-\text { const. } \alpha(1+\Lambda)) \Lambda^{2}\|\Psi\|^{2}-\alpha\|\Psi\|^{2}\left\langle 0\left|E \mathcal{A}^{-1} E^{*}\right| 0\right\rangle \\
- \text { const. } \alpha \Lambda(1+\Lambda)\left(\Psi, H_{f} \Psi\right)-\text { const. } \alpha \Lambda\|p \Psi\|^{2} .
\end{array}
$$

By our a priori estimates (3.21) and (3.26) we prove the theorem.

\section{Proof of Theorem 2}

It suffices to use a cleverly chosen trial wave function. We assume that $\phi(x) \in \mathcal{L}^{2}\left(\mathbb{R}^{3}\right)$ is the ground state of $p^{2}+V$, i.e.

$$
\left(p^{2}+V\right) \phi=-e_{0} \phi \text {. }
$$

Recall,

$$
E^{*} \phi(x, \eta)=\phi(x) \otimes\left[E^{*}(x)|0\rangle\right](\eta), \quad D^{*} p \phi=\sum_{i=1}^{3} p_{x}^{i} \phi(x) \otimes\left[D_{i}^{*}|0\rangle\right](\eta)
$$

are 1-photon functions depending on $x$ and the relative coordinates $\xi-x=$ $\eta$. Therefore, in configuration space, where $\psi(x, \eta)$ denotes one of these functions, we have (cf. the previous section)

$$
\left[\left(p^{2}+H_{f}\right) \psi\right](x, \eta)=\left(\left[p_{x}-p_{\eta}\right]^{2}+\left|p_{\eta}\right|\right) \psi(x, \eta)
$$

For sake of convenience we define the operator

$$
A_{V}=p_{x}^{2}+p_{\eta}^{2}+H_{f}+V+e_{0}=\left(p_{x}^{2}+V+e_{0}\right) \otimes \mathbb{I}+\mathbb{I} \otimes\left(p_{\eta}^{2}+H_{f}\right),
$$

which acts on $\mathcal{L}^{2}\left(\left(\mathbb{R}^{3} ; \mathbb{C}^{2}\right) \otimes\left(\mathbb{R}^{3} ; \mathbb{C}^{2}\right)\right)$ and is obviously positive and invertible.

Now, we choose our trial wave function $\Psi \in \mathcal{H}$ as

$$
\Psi=\left\{\bar{\phi}(x),-2 \sqrt{\alpha} A_{V}^{-1} D^{*} p \bar{\phi}(x, \eta)-\sqrt{\alpha} A_{V}^{-1} \sigma E^{*} \bar{\phi}(x, \eta), 0,0, \ldots\right\},
$$

where $\bar{\phi}=\phi \uparrow$ and $\eta=\xi-x$. We assume $\|\phi\|=1$ and for simplicity we denote the 1-photon part of $\Psi$ as $\psi_{1}$. Notice, $\sigma E^{*} \bar{\phi}(x, \eta)=\phi(x) \otimes(\sigma \uparrow) E^{*}|0\rangle(\eta)$.

First, observe that $A_{V} \bar{\phi}=0$ yields

$$
A_{V} \sigma E^{*} \bar{\phi}=\left(p_{\eta}^{2}+H_{f}\right) \sigma E^{*} \bar{\phi}
$$

Therefore, since $A_{V}$ and $p_{\eta}^{2}+H_{f}$ commute by definition, we infer

$$
A_{V}^{-1} \sigma E^{*} \bar{\phi}=\left(p_{\eta}^{2}+H_{f}\right)^{-1} \sigma E^{*} \bar{\phi}
$$

and

$$
\left(\bar{\phi}, \sigma E A_{V}^{-1} \sigma E^{*} \bar{\phi}\right)=\|\phi\|^{2}\left\langle 0\left|E\left[p_{\eta}^{2}+H_{f}\right]^{-1} E^{*}\right| 0\right\rangle .
$$


Thus, we evaluate

$$
\begin{aligned}
& \left(\Psi, \mathbf{H}_{\alpha} \Psi\right)=\alpha \pi^{-1} \Lambda^{2}\|\Psi\|^{2}-e_{0}\|\Psi\|^{2}+\left(\psi_{1}, A_{V} \psi_{1}\right) \\
& +2 \sqrt{\alpha} \Re\left(\left(\sigma E^{*}+2 D^{*} p\right) \bar{\phi}, \psi_{1}\right)+2 \alpha\left(\psi_{1}, D^{*} D \psi_{1}\right)+2\left(\psi_{1}, p_{x} \cdot p_{\eta} \psi_{1}\right) .
\end{aligned}
$$

Notice, the cross term

$$
\Re\left(\bar{\phi}, \sigma E A_{V}^{-1} D^{*} p \bar{\phi}\right)=\Re\left(\bar{\phi}, \sigma E\left[p_{\eta}^{2}+H_{f}\right]^{-1} D^{*} p \bar{\phi}\right)
$$

vanishes as in the previous section, because it is purely imaginary. Therefore, by our choice of $\psi_{1}$ we get

$$
\begin{aligned}
& \left(\psi_{1}, A_{V} \psi_{1}\right)+2 \sqrt{\alpha} \Re\left(\left(\sigma E^{*}+2 D^{*} p\right) \bar{\phi}, \psi_{1}\right) \\
& \quad=-4 \alpha\left(D^{*} p_{x} \phi, A_{V}^{-1} D^{*} p_{x} \phi\right)-\alpha\|\phi\|^{2}\left\langle 0\left|E\left[p_{\eta}^{2}+H_{f}\right]^{-1} E^{*}\right| 0\right\rangle .
\end{aligned}
$$

Moreover, we have

$$
\begin{aligned}
& \left(\psi_{1}, p_{x} \cdot p_{\eta} \psi_{1}\right)=\alpha\left(D^{*} p_{x} \phi, A_{V}^{-1} p_{x} \cdot p_{\eta} A_{V}^{-1} D^{*} p_{x} \phi\right) \\
& -\alpha\left(\sigma E^{*} \bar{\phi},\left[p_{\eta}^{2}+H_{f}\right]^{-1} p_{x} \cdot p_{\eta}\left[p_{\eta}^{2}+H_{f}\right]^{-1} \sigma E^{*} \bar{\phi}\right) \\
& \quad+2 \alpha \Re\left(D^{*} p_{x} \bar{\phi}, A_{V}^{-1} p_{x} \cdot p_{\eta} A_{V}^{-1} \sigma E^{*} \bar{\phi}\right)
\end{aligned}
$$

The first term in the r.h.s. vanishes by integrating over the $\eta$-variable (the best way to see it is using the representation in momentum space), due to (1.6) and the fact that the operator $A_{V}$ commutes with reflection $\eta \rightarrow-\eta$, respectively $k \rightarrow-k$. The second term vanishes when integrating over the $x$-variable (notice, $\left(\phi, p_{x} \phi\right)=0$ ), and the third term vanishes, because it is again purely imaginary.

Furthermore, since $D A_{V}^{-1} \sigma E^{*} \bar{\phi}=0$ (see (13.17) $)$, we realize, after straightforward calculation, that

$$
\alpha\left(\psi_{1}, D^{*} D \psi_{1}\right) \leq \text { const. } \alpha^{2} \ln (1+\Lambda)^{2} .
$$

Using above estimates and the fact that

$$
\alpha \pi^{-1} \Lambda^{2}-\alpha\left\langle 0\left|E\left[p_{\eta}^{2}+H_{f}\right]^{-1} E^{*}\right| 0\right\rangle=2 \alpha \pi^{-1}[\Lambda-\ln (1+\Lambda)]
$$

we infer

$$
\begin{aligned}
& \left(\Psi, \mathbf{H}_{\alpha} \Psi\right) /(\Psi, \Psi) \leq-e_{0}+2 \alpha \pi^{-1}[\Lambda-\ln (1+\Lambda)] \\
& \quad-4 \alpha\left(D^{*} p_{x} \phi, A_{V}^{-1} D^{*} p_{x} \phi\right)+\mathcal{O}\left(\alpha^{2}\right) \ln (1+\Lambda)\left(\Lambda^{2}+\ln (1+\Lambda)\right)
\end{aligned}
$$

which proves the first statement of the theorem with

$$
\mathcal{E}(V, \Lambda)=4 \alpha\left(D^{*} p_{x} \phi, A_{V}^{-1} D^{*} p_{x} \phi\right) .
$$

The second statement follows by the observation that $\left(D^{*} p_{x} \phi, A_{V}^{-1} D^{*} p_{x} \phi\right)$ is strictly larger than 0 , which is a consequence of the fact that $D^{*} p_{x} \phi$ is a not identically vanishing function $\in \mathcal{L}^{2}\left(\mathbb{R}^{3} \otimes\left(\mathbb{R}^{3}, \mathbb{C}^{2}\right)\right)$ and $A_{V}$ an invertible operator. 


\section{Computation of COncRete numbers}

5.1. Error for the self-energy. We are going to calculate the error $\mathcal{E} r r$ of the self-energy,

$$
\left|\Sigma_{\alpha}-2 \alpha \pi^{-1}[\Lambda-\ln (1+\Lambda)]\right| \leq \mathcal{E} r r\left(\alpha^{2}\right) .
$$

First, it is important to estimate the kinetic energy term $\|p \Psi\|^{2}$ for an approximate ground state $\Psi$ with quite good constants. Since we will calculate anyway with values $\Lambda \leq 1$ we can assume $(\Psi, T \Psi) \leq 2 \alpha \pi^{-1} \Lambda$.

By means of (3.22), (3.23) and then applying (3.25) we obtain

$$
\begin{gathered}
2 \alpha \pi^{-1} \Lambda \geq(\Psi, T \Psi) \geq(1-a)\|p \Psi\|^{2}+\left(\Psi, H_{f} \Psi\right) \\
-4 a^{-1} \alpha\|D \Psi\|^{2}-c \alpha \Lambda^{3}-1 /\left(c \Lambda^{3}\right)\|E \Psi\|^{2} \\
\geq(1-a)\|p \Psi\|^{2}-c \alpha \Lambda^{3}+\left[1-a^{-1} 8 \pi^{-1} \alpha \Lambda-c^{-1} \frac{2}{3 \pi}\right]\left(\Psi, H_{f} \Psi\right)
\end{gathered}
$$

where $1>a>0$. We require the last term [..] to be $\geq 0$. For simplicity, we choose $c=\frac{2}{\pi}$, then our first condition on $\alpha$ reads

$$
\alpha \leq \frac{a \pi}{4 \Lambda}
$$

and additionally

$$
\|p \Psi\|^{2} \leq \frac{2 \alpha \Lambda\left(1+\Lambda^{2}\right)}{\pi(1-a)} .
$$

The main contribution to $\mathcal{E} r r$ stems from the the terms (3.47) and (3.49). In fact the the third diagonal term (3.48) is negligible compared to (3.47) and (3.49).

Evaluating the corresponding integrals yields

$$
\text { (3.49) } \leq 2 \alpha \pi^{-1} \Lambda\|p \Psi\|^{2}+2 \alpha \pi^{-1} \Lambda\left(\Psi, H_{f} \Psi\right),
$$

and

$$
\left(\underline{3.43)}+(\underline{3.46}) \leq \frac{8}{3 \pi} \alpha \Lambda\|p \Psi\|^{2}+\frac{3}{2 \pi} \alpha \Lambda^{2}\left(\Psi, H_{f} \Psi\right) .\right.
$$

In order to incorporate the off-diagonal terms we, for simplicity, double the so gained value for $\mathcal{E} r r$ and derive

$$
\begin{aligned}
\mathcal{E} r r & \leq 2\left[\frac{14}{3 \pi} \alpha \Lambda\|p \Psi\|^{2}+\frac{7}{2 \pi} \alpha \Lambda^{2}\left(\Psi, H_{f} \Psi\right)\right] \\
& \leq \alpha^{2}\left[\frac{56 \Lambda^{2}\left(1+\Lambda^{2}\right)}{3 \pi^{2}(1-a)}+14 \pi^{2} \Lambda^{2}\right]
\end{aligned}
$$

where we used (3.21) and (3.26). 
5.2. Radiative correction. We consider an electron in the field of a nucleus with charge $Z$, i.e.

$$
V=-\frac{Z \beta}{|x|}
$$

where $\beta$ is the "real" fine structure constant $\beta=1 / 137$. The ground state energy of the corresponding Schrödinger operator $p^{2}+V$ is known to be

$$
\text { inf spec }\left[p^{2}+V\right]=-e_{0}=-\frac{1}{4}(\beta Z)^{2} .
$$

The corresponding radiative correction, obtained in (4.15), is given by

$$
\mathcal{E}(V, \Lambda)=-\alpha 4\left(\phi, p D A_{V}^{-1} p D^{*} \phi\right),
$$

where $\phi=\phi(|x|)$ denotes the ground state of $p^{2}+V$. We know

$$
\nabla \phi(|x|)=\partial_{r} \phi(r) \vec{e}_{r}(\theta, \varphi)=e_{0}^{1 / 2} \phi(r) \vec{e}_{r}(\theta, \varphi)
$$

when using polar coordinates. Denote with $\phi_{i}$ the eigenstate of $p^{2}+V$ with corresponding eigenvalue $-e_{i}$. Then, by means of (5.10), we obtain, by straightforward computations,

$$
\left(\phi, p D A_{V}^{-1} p D^{*} \phi\right) \geq 4 \pi e_{0} \sum_{i \geq 1}\left|c_{i}\right|^{2} \int_{0}^{\Lambda} \frac{p}{e_{0}-e_{i}+p^{2}+p} d p \equiv e_{0} F(\Lambda),
$$

where

$$
c_{i}=\int \overline{\phi_{i}(r, \theta, \varphi)} \phi(r) \cos (\theta) r^{2} d r d \Omega
$$

with $d \Omega=\sin \theta d \theta d \varphi$, and the sum runs over all hydrogen eigenstates. Notice, due to textbooks $\sum\left|c_{i}\right|^{2} \sim 2 / 15=\frac{2}{3} \frac{1}{5}$, which in physicists' words is expressed by "80 percent of the average excitation energy $\left\langle e_{0}-e_{n}\right\rangle_{\mathrm{AV}}$ is achieved by the continuous spectrum". Moreover,

$$
\ln [1+\Lambda] / \int_{0}^{\Lambda} \frac{p}{e_{0}-e_{i}+p^{2}+p} d p \rightarrow 1
$$

as $\Lambda \rightarrow \infty$. That is why, for simplicity, we take $\frac{8 \pi}{15} \ln [1+\Lambda]$ to evaluate $F(\Lambda)$ and obtain an approximative radiative correction

$$
R_{C}=\alpha e_{0} \frac{32 \pi}{15} \ln [1+\Lambda]
$$

for the binding energy. (Indeed, for $\Lambda \sim 1$ these two functions perfectly coincide)

5.3. Calculating concrete values of $\alpha$ and $\Lambda$. We search for values of $\alpha$ and $\Lambda$, that guarantee the error of the self energy $\mathcal{E} r r$ being smaller than the radiative correction $R_{C}$. This leads to the condition:

$$
\alpha \leq \operatorname{Min}\left\{\frac{\frac{16 \pi}{15} e_{0} \ln [1+\Lambda]}{\frac{28 \Lambda^{2}\left[1+\Lambda^{2}\right]}{3 \pi^{2}(1-a)}+\frac{7}{\pi^{2}} \Lambda^{2}}, \frac{a \pi}{4 \Lambda}\right\} .
$$


Set $\Lambda=1 / 4$ which corresponds to a photon energy $m c^{2} / 2$, that is an enormously high value compared to the binding energy $e_{0}$.

Therefore, the condition on the coupling parameter, such that the radiative correction $R_{C}$ dominates the error $\mathcal{E} r r$, is given by

$$
\alpha \leq 0.85(\beta Z)^{2} \text {. }
$$

Asking for the nuclear charge numbers $Z$ that guarantees enhanced binding in the physical case $\alpha=1 / 137$, leads to $Z \geq 13$.

However, in the case of smaller values of $\Lambda \sim e_{0}$, which seems physically reasonable, we are in a perfect shape and the error of the self-energy is by far negligible compared to the energy shift.

\section{Appendix A. Auxiliary lemmas}

LEMMA 1. Let $\Psi \in \mathcal{H}$. Then

$$
\begin{aligned}
\left(\Psi, D D\left[p^{2}+H_{f}\right]^{-1} D^{*} D^{*} \Psi\right) \leq\langle & \left.0\left|D D\left[p^{2}+H_{f}\right]^{-1} D^{*} D^{*}\right| 0\right\rangle\|\Psi\|^{2} \\
& +\operatorname{const.\Lambda }\left(\left(\Psi, H_{f} \Psi\right)+\|p \Psi\|^{2}\right) .
\end{aligned}
$$

Proof. We fix an arbitrary photon number $n$. Recall,

$$
\begin{aligned}
\mathcal{F}\left[D^{*} D^{*} \psi_{n}\right]_{n+2}=\frac{1}{\sqrt{(n+2)(n+1)}} \sum_{j=1}^{n+2} \sum_{i=1}^{n+1} G\left(k_{j}\right) \cdot G\left(k_{i}\right) \times \\
\times \psi_{n}\left(l, k_{1}, \ldots, \not k_{j}, \ldots, k_{i}, \ldots, k_{n+2}\right),
\end{aligned}
$$

where $k_{j}$ indicates that the $j$-th variable is omitted. By permutational symmetry we can distinguish between three different terms,

$$
\left(\psi_{n}, D D\left[p^{2}+H_{f}\right]^{-1} D^{*} D^{*} \psi_{n}\right)=I_{n}+I I_{n}+I I I_{n}
$$

First, the diagonal part,

$$
I_{n}=\sum_{\lambda, \nu=1,2} \int \frac{\left[G^{\lambda}\left(k_{1}\right) \cdot G^{\nu}\left(k_{2}\right)\right]^{2}\left|\psi_{n}\left(l, k_{3}, \ldots, k_{n+2}\right)\right|^{2}}{\left|l-\sum_{i=1}^{n+2} k_{i}\right|^{2}+\sum_{i=1}^{n+2}\left|k_{i}\right|} d l d k_{1} \ldots d k_{n+2} .
$$

If we set $\mathcal{Q}=\left|l-\sum_{i=3}^{n+2} k_{i}\right|^{2}+\left|k_{1}+k_{2}\right|^{2}+\sum_{i=1}^{n+2}\left|k_{i}\right|$ and $b=2\left[l-\sum_{i=3}^{n+2} k_{i}\right]$. $\left[k_{1}+k_{2}\right]$ and use the expansion

$$
\frac{1}{\mathcal{Q}-b}=\frac{1}{\mathcal{Q}}+\frac{1}{\mathcal{Q}} b \frac{1}{\mathcal{Q}}+\frac{1}{\mathcal{Q}} b \frac{1}{\mathcal{Q}-b} b \frac{1}{\mathcal{Q}}
$$


then we again see that the second term vanishes when integrating over $k_{1}, k_{2}$. Therefore, with $\mathcal{Q} \geq\left|k_{1}+k_{2}\right|^{2}+\left|k_{1}\right|+\left|k_{2}\right|$ we arrive at

$$
\begin{aligned}
I_{n} \leq \sum_{\lambda, \nu=1,2}[ & \|\Psi\|^{2} \int \frac{\left[G^{\lambda}\left(k_{1}\right) \cdot G^{\nu}\left(k_{2}\right)\right]^{2}}{\left|k_{1}+k_{2}\right|^{2}+\left|k_{1}\right|+\left|k_{2}\right|} d k_{1} d k_{2} \\
& +4 \int \frac{\left[G^{\lambda}\left(k_{1}\right) \cdot G^{\nu}\left(k_{2}\right)\right]^{2}\left[\left|k_{1}\right|+\left|k_{2}\right|\right]^{2}}{\left[\left|k_{1}+k_{2}\right|^{2}+\left|k_{1}\right|+\left|k_{2}\right|\right]^{2}\left(\left|k_{1}\right|+\left|k_{2}\right|\right)} \times \\
& \left.\times\left|l-\sum_{i=3}^{n+2} k_{i}\right|^{2}\left|\psi_{n}\left(l, k_{3}, \ldots, k_{n+2}\right)\right|^{2} d l d k_{1} \ldots d k_{n+2}\right] \\
& \leq\left\langle 0\left|D D\left[p^{2}+H_{f}\right]^{-1} D^{*} D^{*}\right| 0\right\rangle\left\|\psi_{n}\right\|^{2}+\text { const. } \Lambda\left\|p \psi_{n}\right\|^{2} .
\end{aligned}
$$

For convenience we define the operator $|D|(x)$, which can be regarded as the norm of $D(x)$,

$$
|D|(x)=\sum_{\lambda=1,2} \int\left|G^{\lambda}(k)\right| e^{i k \cdot x} a_{\lambda}(k) d k
$$

$|D|^{*}$ denotes the operator adjoint. Similarly, we can define $|E|(x)$. Obviously, GLL, Lemma A. 4] still holds for the "norm" of $D$ and E, namely

$$
|D|^{*}|D| \leq \frac{2}{\pi} H_{f}, \quad|E|^{*}|E| \leq \frac{2}{3 \pi} H_{f}
$$

that can be proved analogously to (3.51). For the second term, with $p^{2}+$ $H_{f} \geq H_{f}$, we evaluate

$$
\begin{aligned}
I I_{n} \leq & n \sum_{\lambda, \nu=1,2} \int \frac{\left|G^{\lambda}\left(k_{1}\right) \cdot G^{\nu}\left(k_{2}\right)\right|\left|G^{\lambda}\left(k_{1}\right) \cdot G^{\nu}\left(k_{n+2}\right)\right|}{\sum_{i=1}^{n+2}\left|k_{i}\right|} \times \\
& \times\left|\psi_{n}\left(l, k_{3}, \ldots, k_{n+2}\right)\right|\left|\psi_{n}\left(l, k_{2}, \ldots, k_{n+1}\right)\right| d l d k_{1} \ldots d k_{n+2} \\
\leq & \int \frac{\left|G\left(k_{1}\right)\right|^{2}}{\left|k_{1}\right|} d k_{1}\left(\left|\psi_{n}\right|,|D|^{*}|D|\left|\psi_{n}\right|\right) \leq \text { const. } \Lambda^{2}\left(\psi_{n}, H_{f} \psi_{n}\right) .
\end{aligned}
$$

Finally, the term where on one side the indices of the created photons differ completely from the indices on the other side,

$$
\begin{gathered}
I I I_{n} \leq n^{2} \sum_{\substack{\lambda, \nu=1,2\\
}} \int \frac{\left|G^{\lambda}\left(k_{1}\right) \cdot G^{\nu}\left(k_{2}\right)\right|\left|G^{\lambda}\left(k_{n+1}\right) \cdot G^{\nu}\left(k_{n+2}\right)\right|}{\sum_{i=1}^{n+2}\left|k_{i}\right|} \times \\
\quad \times\left|\psi_{n}\left(l, k_{3}, \ldots, k_{n+2}\right)\right|\left|\psi\left(l, k_{1}, \ldots, k_{n}\right)\right| d l d k_{1} \ldots d k_{n+2} \\
\leq\left(\left|\psi_{n}\right|,|D|^{*} H_{f}^{-1 / 2}|D|^{*}|D| H_{f}^{-1 / 2}|D|\left|\psi_{n}\right|\right) \leq \operatorname{const.} \Lambda\left(\psi_{n}, H_{f} \psi_{n}\right),
\end{gathered}
$$

where we used

$$
\sum_{i=1}^{n+2}\left|k_{i}\right| \geq\left|\sum_{i=1}^{n+1}\right| k_{i}||^{1 / 2}\left|\sum_{i=2}^{n+2}\right| k_{i}||^{1 / 2},
$$


the fact that we can write

$$
\left[H_{f}\right]^{-1 / 2} \psi_{n}\left(l, k_{1}, \ldots, k_{n}\right)=\left[\sum_{i=1}^{n}\left|k_{i}\right|\right]^{-1 / 2} \psi_{n}\left(l, k_{1}, \ldots, k_{n}\right),
$$

and the first equation of (A.8).

LEMMA 2. Let $\Psi \in \mathcal{H}$ and fix an arbitrary photon number $n$. Then

$$
\begin{aligned}
& \alpha^{3 / 2}\left(\sigma E^{*} \psi_{n+1},\left[p^{2}+H_{f}\right]^{-1} D^{*} D^{*} \psi_{n}\right) \leq \\
& \quad \leq \text { const. }\left[\alpha^{2}\left(\Lambda+\Lambda^{3}\right)\left\|\psi_{n}\right\|^{2}+\alpha\left(\Lambda+\Lambda^{2}\right)\left(\psi_{n+1}, H_{f} \psi_{n+1}\right)\right] .
\end{aligned}
$$

Proof. Obviously,

$$
\left(\sigma E^{*} \psi_{n+1},\left[p^{2}+H_{f}\right]^{-1} D^{*} D^{*} \psi_{n}\right)=I_{n}+I I_{n},
$$

where

$$
\begin{aligned}
& I_{n} \leq(n+1) \sum_{\lambda, \nu=1,2} \int \frac{\left|H^{\lambda}\left(k_{1}\right)\right|\left|G^{\lambda}\left(k_{1}\right)\right|\left|G^{\nu}\left(k_{2}\right)\right|}{\left|k_{1}\right|} \times \\
& \times\left|\psi_{n}\left(l, k_{3}, \ldots, k_{n+2}\right)\right|\left|\psi_{n+1}\left(l, k_{2}, \ldots, k_{n+2}\right)\right| d l d k_{1} \ldots d k_{n+2} \\
& \leq \text { const. } \Lambda^{2}\left\|\psi_{n}\right\|^{1 / 2} \int\left|G\left(k_{2}\right)\right| \sqrt{\left|k_{2}\right| \rho_{\psi_{n+1}}\left(k_{2}\right)} d k_{2} \\
& \leq \text { const. }\left(\alpha^{1 / 2} \Lambda^{3}\left\|\psi_{n}\right\|^{2}+\alpha^{-1 / 2} \Lambda^{2}\left(\psi_{n+1}, H_{f} \psi_{n+1}\right)\right)
\end{aligned}
$$

For the second term of (A.14) we obtain

$$
\begin{gathered}
I I_{n} \leq(n+1)^{3 / 2} \sum_{\lambda, \nu=1,2} \int \frac{\left|H^{\lambda}\left(k_{1}\right)\right|\left|G^{\lambda}\left(k_{n+1}\right)\right|\left|G^{\nu}\left(k_{n+2}\right)\right|}{\sum_{i=1}^{n+2}\left|k_{i}\right|} \times \\
\times\left|\psi_{n}\left(l, k_{1}, \ldots, k_{n}\right)\right|\left|\psi_{n+1}\left(l, k_{2}, \ldots, k_{n+2}\right)\right| d l d k_{1} \ldots d k_{n+2} \\
\leq(n+1)^{3 / 2} \sum_{\lambda, \nu=1,2} \int \frac{\left|H^{\lambda}\left(k_{1}\right)\right|\left|\psi_{n}\left(l, k_{1}, \ldots, k_{n}\right)\right|}{\left|\sum_{i=1}^{n}\right| k_{i}||^{1 / 2}} \times \\
\times \frac{\left|G^{\lambda}\left(k_{n+1}\right)\right|\left|G^{\nu}\left(k_{n+2}\right)\right|\left|\psi_{n+1}\left(l, k_{2}, \ldots, k_{n+2}\right)\right|}{\left|\sum_{i=2}^{n+1}\right| k_{i}||^{1 / 2}} d l d k_{1} \ldots d k_{n+2} \\
\leq\left\|| E | H _ { f } ^ { - 1 / 2 } | \psi _ { n } | | | | | | D | H _ { f } ^ { - 1 / 2 } \left|D\left\|\psi_{n+1} \mid\right\|,\right.\right.
\end{gathered}
$$

which implies the statement of the lemma by use of (A.8).

Acknowledgment: The author has been supported by a Marie Curie Fellowship of the European Community programme "Improving Human Research Potential and the Socio-economic Knowledge Base" under contract number HPMFCT-2000-00660. Furthermore, he thanks his friend Robert Seiringer 
for many valuable discussions and Semjon A. Vugalter for initiating the study of this problem.

\section{REFERENCES}

[BFS1] V. Bach, J. Fröhlich, I.-M. Sigal Mathematical theory of non-relativistic matter and radiation, Lett. Math. Phys. 34, 183-201 (1995)

[BFS2] V. Bach, J. Fröhlich, I.-M. Sigal Quantum electrodynamics of confined nonrelativistic particles, Adv. Math. 137, 299-395 (1998)

[BFS3] V. Bach, J. Fröhlich, I.-M. Sigal Spectral Analysis for Systems of Atoms and Molecules Coupled to the Quantized Radiation Field, Commun. Math. Phys. 207, 249-290 (1999)

[GLL] M. Griesemer, E.H. Lieb, M. Loss Ground states in non-relativistic quantum electrodynamics, Inventiones Math. 145, 557-595 (2001)

[Hi] F. Hiroshima, Self-adjointness of the Pauli-Fierz Hamiltonian for arbitrary values of coupling constants, Ann. Henri Poincaré 3, 171 (2002).

[HVV] Ch. Hainzl, V. Vougalter, S.-A. Vugalter Enhanced binding in non-relativistic QED, Commun. Math. Phys. 233, 13-26 (2003)

[HS] F. Hiroshima, H. Spohn Enhanced Binding through coupling to a Quantum Field, Ann. Henri Poincaré 21159 (2001)

[LL] E.H. Lieb, M. Loss Self-Energy of Electrons in non-perturbative QED, Differential Equations and Mathematical Physics, University of Alabama, Birmingham, 1999, R. Weikard and G. Weinstein, eds. 279-293, Amer. Math. Soc./Internat. Press (2000). arXiv math-ph/9908020, mp_arc 99-305.

Mathematisches Institut, LMU München, Theresienstrasse 39, 80333 MuNiCH, Germany

E-mail address: hainzl@mathematik.uni-muenchen.de 